A. Pramesh Rao, G. Swarup and Gopal-Krishna, eds.

\title{
Extreme Relic Radio Sources in Four Southern Clusters
}

\author{
H. Andernach
}

Depto. de Astronomía, Univ. de Guanajuato, Guanajuato, Mexico

O.B. Slee

ATNF, CSIRO, PO Box 76, Epping, NSW 2121, Australia

A.L. Roy

MPI für Radioastronomie, Auf dem Hügel 69, D-53121 Bonn, Germany

M. Ehle

MPI für Extraterrestrische Physik, D-85748 Garching, Germany

\begin{abstract}
We describe the highest-resolution radio observations yet made of relic radio sources. These relics have extremely steep $(\alpha \leq-2.5)$ spectra and are located in four southern Abell clusters (A 13, A 85, A133, A 4038). VLA images at $1.4 \mathrm{GHz}$ and $\sim 4$ " resolution show a remarkable variety of fine structure like arcs, wisps, plumes, and loops. Integrated polarization fractions range from $2.3 \%$ to $16.8 \%$. Deep red CCD images do not reveal any optical object with a brightness and/or position typical of a radio host galaxy. The X-ray centroids in ROSAT images tend to be displaced from the cluster potential wells in the direction of the relics in A85, A133 and A4038, suggesting that some of the X-rays may be inverse Compton or excess thermal emission from the relic itself.
\end{abstract}

A small fraction of rich galaxy clusters contain a diffuse steep-spectrum source with no optical counterpart. Some of these sources take the form of a halo near the cluster centre, while others, with various morphologies, are situated closer to the cluster perimeter and are termed "relics". Although relics were generally thought to be amorphous blobs of relaxed radio plasma without ongoing electron reacceleration, Slee \& Roy (1998) found much fine structure in a high resolution ( $\left.4^{\prime \prime}\right)$ image of the relic in A4038.

We present further higher-resolution observations with the VLA of the known radio relics A13_6a/b/c, A85_25a/b/c and A133_7a/b, and further measurements from the images of A4038_9 (Slee \& Roy 1998). These relics were first identified by Slee \& Reynolds (1984) who demonstrated their extremely steep spectra and lack of optical host galaxies. Reobservation with the VLA at $\sim 15^{\prime \prime}$ resolution (Slee et al. 1994) did not give further clues on their origin or optical identification. In $1997 / 98$ we observed the four relics with the VLA at $1.4 \mathrm{GHz}$ at $\sim 4$ " resolution, the highest yet attained for imaging radio relics. The data were self-calibrated and the dirty images were CLEANed with the AIPS task $\mathrm{MX}$, resulting in an rms noise from 17 to $21 \mu \mathrm{Jy}$ beam $^{-1}$. 
Separate images were made for two frequency bands at 1.385 and $1.465 \mathrm{GHz}$. Although closely spaced in frequency, these yielded useful values of the average spectral index, $\alpha\left(\mathrm{S} \sim \nu^{\alpha}\right)$, for the relics (col. 5 of Table 1). Flux densities at other frequencies from the literature were used to plot the broad-band spectra of the relics (insets in Fig. 1). The spectra show a characteristic curvature with pronounced flattening at low frequencies; below $\sim 100 \mathrm{MHz}$, all spectra flatten to $\alpha \geq-1.0$. The intersection of lines fitted to the low- and high-frequency points may define a "break frequency", $\nu_{\mathrm{b}}$, as listed in Table 1. In A133 an additional break occurs at $2.7 \mathrm{GHz}$, where the spectrum steepens to $\alpha<-4.0$.

Average polarization percentages determined from images of polarized intensity are listed in Table 1. The position angle of the E-vector at the source is not known for any of these relics because we lack higher-frequency images to determine the rotation measure. The polarization varies significantly from one relic to another. A85 is highly polarized in some areas at $1.425 \mathrm{GHz}$, implying that the fields are not highly tangled and that Faraday depolarization is low. On the other hand, depolarization in A133 and A4038 is rather high.

A detailed description of the images and their optical overlays can be found in Slee et al. (2000). Fig. 1 shows radio images and spectra of the relics in A85 and A133. The radio images of the relics show a remarkable variety of fine structure in the form of arcs and blobs of enhanced surface brightness. Many of the arcs are barely resolved $\left(5 \mathrm{kpc}, H_{\circ}=75 \mathrm{~km} \mathrm{~s}^{-1} \mathrm{Mpc}^{-1}\right)$ in their transverse directions, but can be up to $100 \mathrm{kpc}$ long. There is no consistent morphology for the relics; in A133 (Fig. 1), four arc-like features appear to begin near the high-brightness centre of the relic, but in A13 and A85 the structures have a variety of orientations. The arcs in A85, for example, undergo large changes in direction, and the loop in the SW structure shows a reversal of $\sim 180^{\circ}$. The relic in A4038 (Slee \& Roy 1998) has a radio morphology similar to that of A85.

Table 1. Integrated parameters of the relics. Columns are: (1) Abell cluster and source name from Slee et al. (1994), (2) largest linear size of the radio relic in kpc, assuming cluster membership, (3) magnitude of the faintest object in our CCD image within the relic's extent, (4) $1.4 \mathrm{GHz}$ flux, (5) integrated spectral index near $1.4 \mathrm{GHz}$, (6) break frequency (see text), (7) integrated polarization of the relic at $1.4 \mathrm{GHz}$.

\begin{tabular}{lrrrrrr}
\hline Name & LLS & $\mathrm{m}_{R}^{L I M}$ & $\mathrm{~S}_{1.425}(\mathrm{mJy})$ & $\alpha(1.4 \mathrm{GHz})$ & $\nu_{\mathrm{b}} / \mathrm{MHz}$ & $\mathrm{p}(\%)$ \\
\hline $\mathrm{A} 13 \_6 \mathrm{a} / \mathrm{b} / \mathrm{c}$ & 261 & 22.4 & $21.1 \pm 2.1$ & $-3.9 \pm 1.2$ & 250 & $11.6 \pm 4.1$ \\
$\mathrm{~A} 85 \_25 \mathrm{a} / \mathrm{b} / \mathrm{c}$ & 150 & 18.9 & $29.7 \pm 3.0$ & $-3.0 \pm 0.8$ & 180 & $16.4 \pm 8.8$ \\
$\mathrm{~A} 133 \_7 \mathrm{a}$ & 56 & 22.0 & $130 . \pm 5$. & $-2.5 \pm 0.5$ & 125 & $2.3 \pm 1.5$ \\
$\mathrm{~A} 4038 \_9$ & 56 & 22.4 & $40.6 \pm 3.0$ & $-2.9 \pm 0.7$ & 300 & $4.6 \pm 2.3$ \\
\hline \hline
\end{tabular}

Our new high-resolution maps, overlaid on deep CDD images, obtained by us with the AAT, do not give further hints on the likely optical counterparts of the relics. The brightest galaxy within the radio contours of the relic in A85 (marked "A" in Fig. 1) has $M_{R}=-21.7$ (in the cluster rest frame) and is therefore bright enough to be a former host for a cluster radio galaxy. However, located in a region of very low brightness near the edge of the radio image, it is an unlikely host. Galaxies B and C, just outside the eastern boundary are 

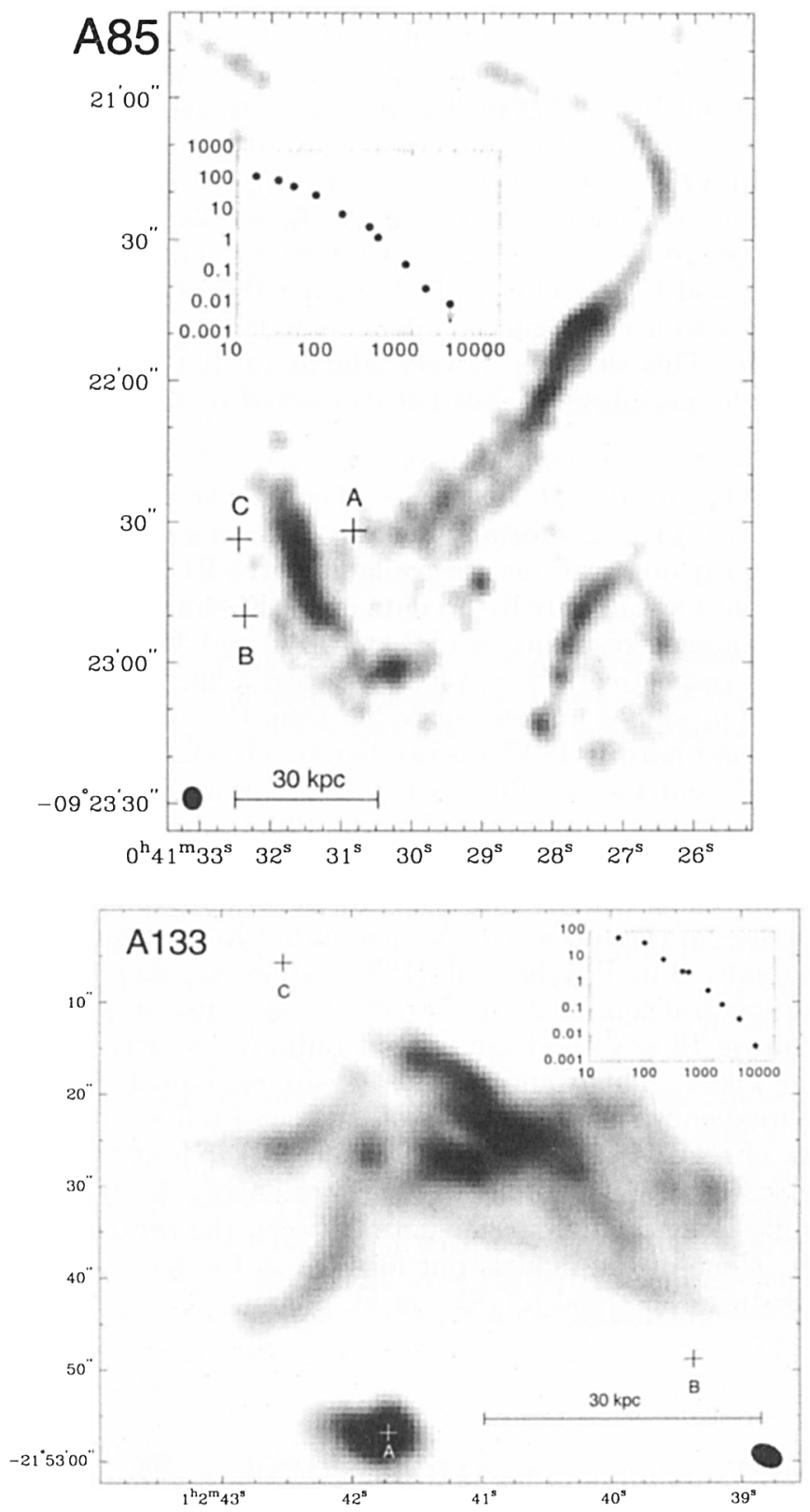

Figure 1. $1.4 \mathrm{GHz}$ grey-scale VLA images of relics in A85 and A133. Filled ellipses in the corners show the FWHP of the restoring beams. Coordinates are for equinox J2000, and labelled plus signs indicate the positions of the brightest galaxies closest to the radio emission regions. Galaxy A in A133 is the central cD. The insets show integrated radio spectra with the abscissa labelled in $\mathrm{MHz}$ and the ordinate in $\mathrm{mJy}$. The horizontal bar indicates the linear scale $\left(H_{\circ}=75 \mathrm{~km} \mathrm{~s}^{-1} \mathrm{Mpc}^{-1}\right)$. 
also bright enough for former hosts, but, if so, they have since moved away from the radio plasma. None of the numerous faint galaxies within the contours is situated at a significant brightness peak. We conclude that the source is a radio relic rather than a conventional radio galaxy. The situation is similar for A13.

For the relic in A133 there is no galaxy with $M_{\mathrm{R}} \leq-14.4$ within the radio contours. The bright $\mathrm{cD}$ galaxy ("A" in Fig. $1, M_{\mathrm{R}}=-24.2$ ) is a separate radio source $\sim 40 "(40 \mathrm{kpc})$ in projection due $S$ of the relic's centroid. The remaining optical galaxies (B and C) are ellipticals, but even the brightest is too faint to have once been host to a radio galaxy. We conclude that the extended source is a true radio relic. This situation is very similar to that in A4038, where the relic is located in the periphery of, but not connected to the central $\mathrm{cD}$ galaxy.

Recent evidence for non-thermal inverse Compton X-ray emission from the Coma cluster halo (Rephaeli et al. 1999, Fusco-Femiano et al. 1999) motivated us to search existing X-ray images for any enhanced X-ray emission from the relic regions. Pointed X-ray observations are available in the ROSAT archive for A85, A133 and A4038, but for A13 only RASS data exist (Ebeling et al. 1996). Several observations were merged resulting in $15.9 \mathrm{ks}$ PSPC and $31.7 \mathrm{ks}$ HRI exposures for A85 and A133, respectively. For A4038 we used a single $3.3 \mathrm{ks} \mathrm{PSPC} \mathrm{data}$ set. From these we produced images out to $20^{\prime}$ from the optical axis in the full, as well as the soft and hard ROSAT energy bands. Overlays of radio and X-ray images show slightly enhanced diffuse X-ray emission and/or a shift of the Xray emission centroid towards the relic. For the PSPC observations of A85 and A4038 these asymmetries are most pronounced in the hard energy band, as can be expected for inverse Compton emission which has a steep spectrum, similar to the radio emission of the relics. For A85 enhanced X-ray emission in the relic region was also reported by Bagchi et al. (1998). However, there appears to be a difference in the spectral composition of excess X-ray emission in the Coma halo (detected only above $18 \mathrm{keV}$ ) and the present radio relics, with excess emission in the range $0.5-2 \mathrm{keV}$. This could be most easily explained with a difference in the very-low-frequency spectral indices of halos and relics: relics could have a higher density of relativistic electrons radiating at the lower frequencies that most efficiently scatter CMB photons to the lower energy X-ray spectral band.

The four relics seem to be intermediate between the central halo- and the edge-type relics. None of the models put forward in the past seem to describe adequately all features observed (cf. Slee et al. (2000).

\section{References}

Bagchi, J., Pislar, V., \& Lima Neto, G.B. 1998, MNRAS, 296, L23

Ebeling, H., et al. 1996, MNRAS, 281, 799

Fusco-Feminano, R., et al. 1999, ApJ, 513, L21

Rephaeli, Y., Gruber, D., Blanco, P. 1999, ApJ, 511, L21

Slee, O.B., \& Reynolds, J.E., 1984, PASAu, 5, 516

Slee, O.B., Roy, A.L., \& Savage, A. 1994, Aust. J. Phys., 47, 145

Slee, O.B., \& Roy, A.L. 1998, MNRAS, 297, L86

Slee, O.B., \& Roy, A.L., Andernach, H., \& Ehle, M. 2000, AJ, submitted 